\title{
Ultrathin NbN Films for Superconducting Single-Photon Detectors
}

\author{
W. SŁysz ${ }^{a *}$, M. Guziewicz ${ }^{a}$, M. Borysiewicz ${ }^{a}$, J.Z. DomagaŁa ${ }^{b}$, I. PASternak ${ }^{a}$, \\ K. Hejduk ${ }^{a}$, W. RzOdKIEWicz ${ }^{a}$, J. RAtajCzAK ${ }^{a}$, J. BAR ${ }^{a}$, M. WęGRZECKi $^{a}$, \\ P. Grabiec ${ }^{a}$, R. Grodecki ${ }^{a}$, I. Węgrzecka ${ }^{a}$ And R. Sobolewski ${ }^{a, c}$ \\ ${ }^{a}$ Institute of Electron Technology, al. Lotników 32/46, 02-668 Warsaw, Poland \\ ${ }^{b}$ Institute of Physics, Polish Academy of Sciences, al. Lotników 32/46, 02-668 Warsaw, Poland \\ ${ }^{c}$ Departments of Electrical and Computer Engineering and Physics and Astronomy, Materials Science Program \\ and Laboratory for Laser Energetics, University of Rochester, Rochester, NY-14627-0231, USA

\begin{abstract}
We present our research on fabrication and structural and transport characterization of ultrathin superconducting $\mathrm{NbN}$ layers deposited on both single-crystal $\mathrm{Al}_{2} \mathrm{O}_{3}$ and $\mathrm{Si}$ wafers, and $\mathrm{SiO}_{2}$ and $\mathrm{Si}_{3} \mathrm{~N}_{4}$ buffer layers grown directly on Si wafers. The thicknesses of our films varied from $6 \mathrm{~nm}$ to $50 \mathrm{~nm}$ and they were grown using reactive $\mathrm{RF}$ magnetron sputtering on substrates maintained at the temperature $850{ }^{\circ} \mathrm{C}$. We have performed extensive morphology characterization of our films using the X-ray diffraction method and atomic force microscopy, and related the results to the type of the substrate used for the film deposition. Our transport measurements showed that even the thinnest, $6 \mathrm{~nm}$ thick NbN films had the superconducting critical temperature of 10-12 K, which was increased to $14 \mathrm{~K}$ for thicker films.
\end{abstract}

PACS: 74.62.Bf, 74.78.-w, 81.15.Cd, 81.15.Jj

\section{Introduction}

Superconducting single photon detectors (SSPD) are novel optoelectronic devices that are currently subject of intensive research and development. The SSPDs are typically meander-type nanostructures consisting of up to $0.5 \mathrm{~mm}$ long and approximately $100 \mathrm{~nm}$ wide stripes patterned in an ultrathin superconducting $\mathrm{NbN}$ (NbTiN) film. They operate below the NbN superconducting critical temperature $T_{\mathrm{c}}$ and are biased on with a subcritical current [1]. The absorption of a photon generates a hot spot that grows until a resistive region is formed across the nanostripe, thus, producing a detectable voltage pulse. Single photons with wavelengths ranging from infrared $(\approx 2 \mu \mathrm{m})$ to ultraviolet $(<400 \mathrm{~nm})$ can be detected with efficiencies reaching $57 \%$ [2].

Main properties of SSPDs that make them so attractive for various photon-counting applications are: relatively high quantum efficiency (QE) in visible-light and near-infrared wavelengths, negligibly low dark-count levels, picosecond pulse-to-pulse timing jitter and ultrahigh (up to $\mathrm{GHz}$ ) counting rate [3]. SSPDs are expected to play a dominant role in such applications as optical quantum information processing [4], satellite communications [4], and medical diagnostics [5].

Niobium nitride is a material of choice for manufacturing of SSPDs, thanks to its stable superconducting properties at relatively high temperatures even in the ultrathin stripe form $\left(T_{\mathrm{c}}=16-18 \mathrm{~K}\right.$ for bulk material) [6]

* corresponding author; e-mail: wslysz@ite.waw.pl and already well established technological procedures for the ultrathin-film growth and processing. SSPDs are typically made by patterning a meander-type nanostructure in an ultrathin $(\approx 5 \mathrm{~nm})$ film deposited on a sapphire [1], $\mathrm{MgO}$ [7], or, recently, 3C-SiC/Si [8] single-crystal substrates. Single-crystal quality $\mathrm{NbN}$ films are deposited by either DC or RF reactive magnetron sputtering on substrates maintained at the $700-850^{\circ} \mathrm{C}$ temperature range $[1,9]$. According to literature, $\mathrm{NbN}$ growth on the $\mathrm{MgO}$ substrate results in the best values of $T_{\mathrm{c}}$, e.g., $T_{\mathrm{c}}=12 \mathrm{~K}$ for a $4 \mathrm{~nm}$ thick film [10].

Currently proposed new SSPD receivers directly integrated with advanced optoelectronic structures, such as distributed Bragg reflectors, optical waveguides, or plasmonic nano-antennas, require, however, the new optoelectronics-compatible substrates. We present here our investigations on technology of the NbN thin-film growth using reactive magnetron sputtering deposition on different optical substrates, varying from $\mathrm{Al}_{2} \mathrm{O}_{3}$ through $\mathrm{Si}$ to $\mathrm{SiO}_{2}$ and $\mathrm{Si}_{3} \mathrm{~N}_{4}$. This work presents structural characterization of ultrathin NbN films using both the X-ray diffraction (XRD) and high-resolution X-ray diffraction (HRXRD) methods. Texture of the films is evidenced by the XRD spectra [11], while the defect structure is visualized using the reciprocal lattice maps of main Bragg reflexes. Surface morphology of our ultrathin films was imaged by atomic force microscopy (AFM). Finally, the sample superconducting properties such as $T_{\mathrm{c}}$ were studied by electrical transport measurements and directly correlated with structural characteristics. 


\section{Experimental}

NbN films were grown using a high-temperature reactive RF magnetron sputtering system $\gamma 1000 \mathrm{C}$ made by the Surrey Nanosystems Ltd. The films were deposited from a 3-inch diameter $\mathrm{Nb}$ target at pulsed $\mathrm{DC}$ power of $220 \mathrm{~W}$ in $\mathrm{N}_{2}-\mathrm{Ar}$ gas mixture at pressure of $8 \mu$ bar, and at controlled substrate temperature in range from $700{ }^{\circ} \mathrm{C}$ to $850{ }^{\circ} \mathrm{C}$. We used $\mathrm{Al}_{2} \mathrm{O}_{3}$ (0001), $\mathrm{Si}(001)$, as well as $\mathrm{SiO}_{2} / \mathrm{Si}$ and $\mathrm{Si}_{3} \mathrm{~N}_{4} / \mathrm{Si}$ wafers as substrates. In the last two cases, our $\mathrm{NbN}$ films were deposited on typically $100 \mathrm{~nm}$ thick, thermal-grown $\mathrm{SiO}_{2}$ or $\mathrm{Si}_{3} \mathrm{~N}_{4}$ deposited by the chemical vapor deposition (CVD) method. High purity of the films was achieved due to the $4 \mathrm{~N} 5$ purity of the target and $6 \mathrm{~N}$ purity of the process gases, as well as maintaining the background pressure of $10^{-8}$ mbar. Thickness of the NbN film was controlled by the deposition time and it was chosen to cover the $6-50 \mathrm{~nm}$. The accurate thickness was calculated based on a simulation of the ultrathin film XRD spectrum. Moreover, structure of the $\mathrm{NbN}$ film was modified by post-deposition rapid annealing (RTP) in $\mathrm{Ar}$ at temperature of $1000^{\circ} \mathrm{C}$ for $20 \mathrm{~min}$.

Thickness of the NbN film was controlled by the deposition time and it was chosen in range of 6-50 nm. The accurate thickness was calculated from simulation of XRD spectrum of the ultrathin film. Characterization of structural properties of the $\mathrm{NbN}$ films was performed by analysis of X-ray diffraction $2 \theta / \omega$ patterns collected in a full angular range employing Philips X'Pert MPD diffractometer working in a configuration with an incident-beam Johansson monochromator and a semiconductor strip detector. For HRXRD analysis the Philips X'Pert MRD diffractometer equipped with the X-ray mirror, $\mathrm{Cu} K_{\alpha}$ Bartels type monochromator, and 3-bounce analyzer in reflected beam line was applied.

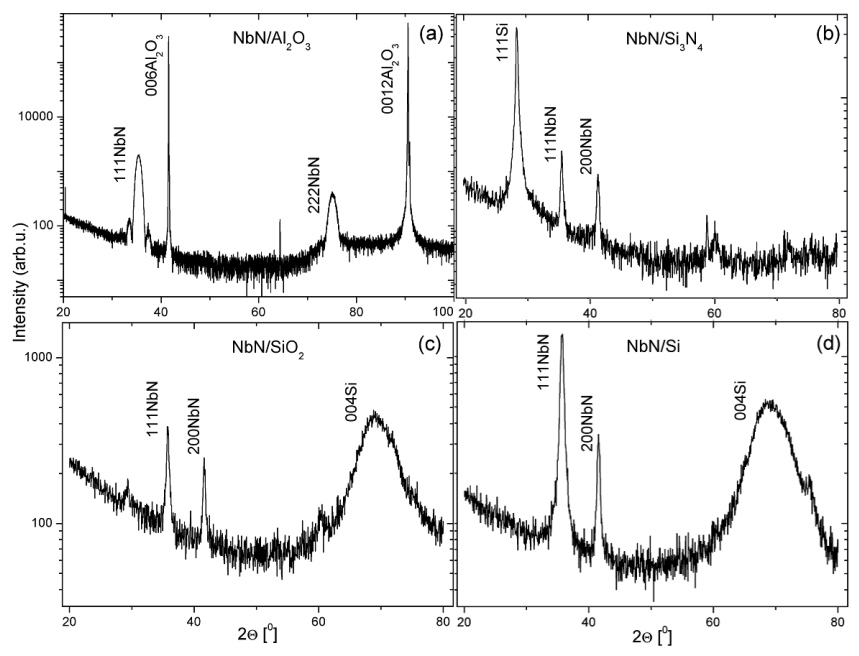

Fig. 1. X-ray diffraction patterns; $2 \theta / \omega$ scans for $\mathrm{NbN}$ films on substrates: (a) sapphire(0001), (b) $\mathrm{Si}(111) /$ $\mathrm{Si}_{3} \mathrm{~N}_{4}$, (c) $\mathrm{Si}(001) / \mathrm{SiO}_{2}$, (d) $\mathrm{Si}(001) / \mathrm{Si}_{3} \mathrm{~N}_{4}$.

\section{Films properties}

\subsection{X-ray (XRD) analysis}

Figure 1a shows a $2 \theta / \omega$ scan for a $6 \mathrm{~nm}$ thick $\mathrm{NbN}$ wafer deposited on sapphire substrate. We can see the peaks from first- and second-order reflex from (111) planes of $\mathrm{NbN}$. The absence of peaks from any other planes inclined to the (111) NbN planes confirmed the epitaxial nature (high structural perfection) of the deposited films. The further examination of the crystalline quality of our $\mathrm{NbN}$ films deposited on sapphire was done by HRXRD method. Patterns shown in Fig. 1b, c and d, collected for $\mathrm{NbN}$ films deposited on $\mathrm{SiO}_{2}, \mathrm{Si}_{3} \mathrm{~N}_{4}$, and $\mathrm{Si}$ substrates, respectively, contain $\mathrm{NbN}$ reflections of the same type from peaks 111 and 200 . It confirms the polycrystalline nature of the films. Absence of other $h k l$ peaks indicates that the polycrystalline films on $\mathrm{Si}$ are textured. Crystalline grains are preferentially oriented in the out-of-plane $\langle 111\rangle$ and $\langle 100\rangle$ direction, but they are random in-plane directions.

\section{2. $H R X R D$}

The analysis of the quality of our films was also carried out based on the reciprocal lattice maps (RLM) of the 111 and 311 reflexes. Patterns of the 111 reflexes are presented in Fig. 2 and they show a perfect dependence of the intensity changes versus the reflex angle $2 \theta$. A wide range of oscillation peaks (in literature called "Pendellösung") visible in Fig. 2 indicates a very sharp and flat interface. The sample after annealing at $1000^{\circ} \mathrm{C}$ was characterized by a perfect crystalline quality of the (111) planes confirmed by wide range of thickness oscillations visible in Fig. 2b, as well as by the 111 RLM (not shown) and a simple $\omega$ scan (rocking curve (RC)) collected with analyzer and shown in Fig. 3a. The value of $11^{\prime \prime}$ for the full-width-at-half-maximum (FWHM) of the RC of the $111 \mathrm{NbN}$ reflex indicates a very good crystallinity of the film. Such low FWHM value is typically attained only on excellent single crystals for example $\mathrm{Si}$, using high-resolution diffractometer. The latter means absence of a mosaics tilt. A shape of the diffraction signal for the annealed sample (Fig. 3b) is not symmetrical like for the as-deposited one, which can be caused by a worse geometry of the sample, having a size smaller than the beam spot.

In Table, we present values of a lattice constant $a$ calculated for two reflexes assuming a cubic lattice unit. This is a simple method to check if the unit is distorted. The results confirm the cubic lattice of our $\mathrm{NbN}$ films, as well that it is relaxed. The $a$ values are lower than reported in literature $(a=4.389 \AA[8])$ that may be caused by difference in stoichiometry. The problem requires an investigation to exclude residual gases being trapped during the film deposition.

The changes of diffuse scattering (wide isocontours ellipses around the 311 point) collected for the 311 RLM for an exemplary film are shown in Fig. 4. Both maps 


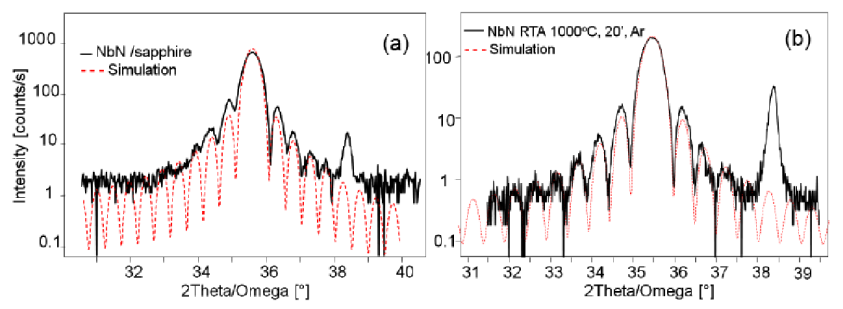

Fig. 2. The $2 \theta / \omega$ experimental scan (solid line) of the 111 Bragg reflex and simulation (dotted line) from an $18 \mathrm{~nm} \mathrm{NbN}$ film deposited on sapphire (0001): (a) before annealing, (b) after annealing.
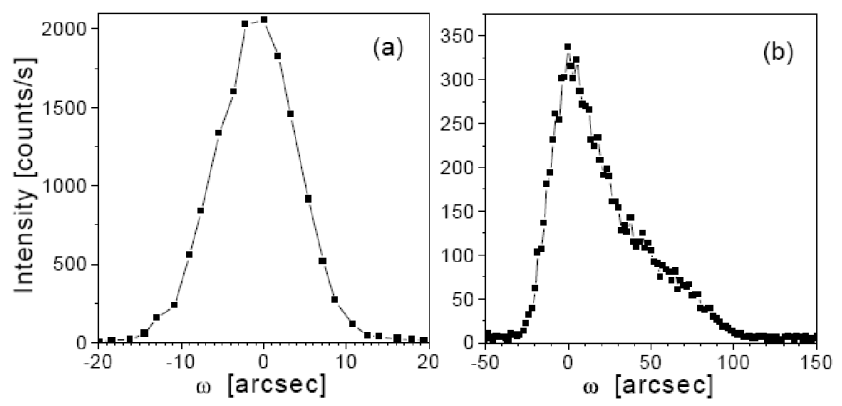

Fig. 3. Rocking curve for an $18 \mathrm{~nm} \mathrm{NbN}$ film deposited on sapphire (0001): (a) before annealing, (b) after annealing.

are of small intensity and were obtained using a slit before the detector. Figure 4a shows a very regular circle map with four times smaller intensity than for the map in Fig. 4b. It is in the contradiction to the intensities of corresponding 111 reflexes. The latter indicates that mosaics twist for the sample before annealing is bigger. An elongated ellipse seen in Fig. 4b looks like an image of a perfect thin layer. Small, but still visible two circles placed on opposite sides of the main ellipse are equivalent to the "Pendellösung" fringes from Fig. 2.

Based on information presented in Figs. 2, 3, and 4 we can conclude that annealing at $1000^{\circ} \mathrm{C}$ strongly influenced the crystalline quality of our films. Specifically in annealed films mosaics tilt seems to be slightly stronger, although it is close to the experimental error. On the other hand, the mosaics twist becomes lower. Finally, the lattice constant $a$ (Table) after annealing is getting bigger.

TABLE

The values of the lattice constant $a$ calculated from 2 reflexes assuming cubic lattice unit and the film thickness from simulation of the 111 Bragg reflection.

\begin{tabular}{c|c|c|c}
\hline \hline \multirow{2}{*}{ NbN } & \multicolumn{2}{|c|}{ Lattice constant $a[\AA]$} & \multirow{2}{*}{ Thickness $[\AA]$} \\
\cline { 2 - 3 } & from 111 refl. & from 311 refl. & \\
\hline as-deposited & $4.368 \pm 0.001$ & $4.366 \pm 0.004$ & $18 \pm 1$ \\
after annealing & $4.379 \pm 0.001$ & $4.379 \pm 0.004$ & $17.6 \pm 0.3$
\end{tabular}

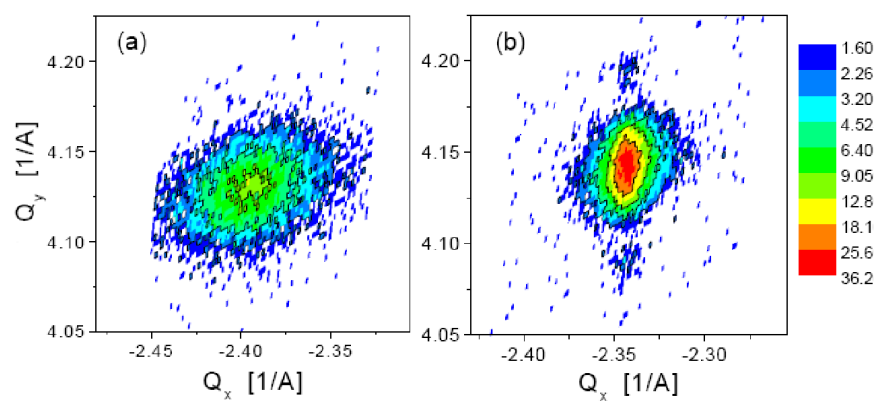

Fig. 4. Reciprocal lattice map of the 311 reflex for an $18 \mathrm{~nm} \mathrm{NbN}$ film deposited on sapphire (0001): (a) before annealing, (b) after annealing. Diagrams represent intensity scale (counts/s). Maxima of intensities are located at centers of the patterns.

\subsection{AFM measurement}

Surface morphology and roughness of our NbN samples were studied using the Veeco Innova SPM AFM instrument. The AFM topographic images $\left(1 \times 1 \mu \mathrm{m}^{2}\right.$ scan size) were taken in a tapping mode. In Fig. 5, we present

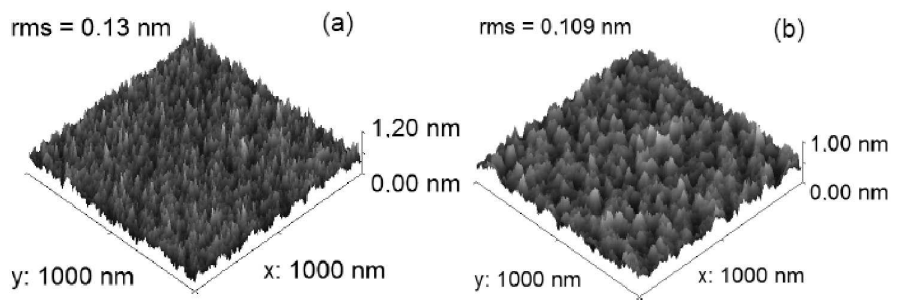

Fig. 5. AFM image of a $9 \mathrm{~nm} \mathrm{NbN}$ film deposited on sapphire: (a) before annealing, (b) after annealing.

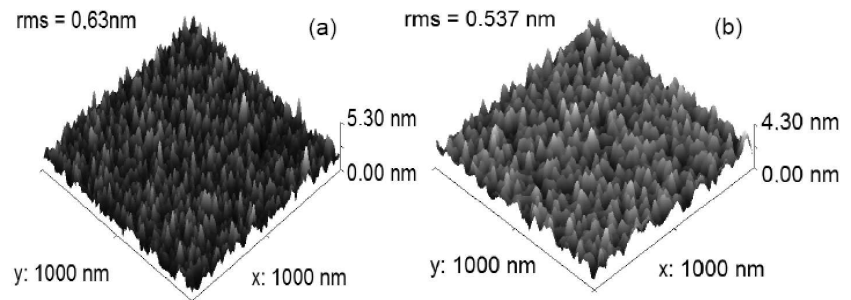

Fig. 6. AFM image of an $18 \mathrm{~nm} \mathrm{NbN}$ film deposited on the $\mathrm{SiO}_{2}$ substrate: (a) before annealing, (b) after annealing.

AFM images of $\mathrm{NbN}$ films deposited on sapphire before and after annealing, while Fig. 6 shows the analogous images, but for $\mathrm{NbN}$ films on $\mathrm{SiO}_{2}$. Comparing the images, we see that for the sapphire substrate, the roughness of the $\mathrm{NbN}$ film is generally lower. In addition, annealing process (Figs. 5b and 6b) improves the film smoothness. Overall, we observed the analogous behavior to that presented in Fig. 6, for all our non-epitaxial, ultrathin films, namely, deposited on $\mathrm{Si}, \mathrm{SiO}_{2}$, and $\mathrm{Si}_{3} \mathrm{~N}_{4}$ substrates. All 
these films were smoother after the RTP process. However, for thicker NbN films (20 nm and above), the effect of RTP was opposite, as we observed rapid worsening of the layer's smoothness after annealing. The latter is due to the growth of columnar defects, which are typical for $\mathrm{NbN}$, and nitrides in general [12].

\subsection{Low temperature resistance}

Measurements of the resistivity $R$ dependence of our NbN films on temperature $T$ were performed using a standard, four-probe technique in a liquid He dewar. The superconducting transition was observed in all studied by us films. Figure 7 a shows the $R$ vs. $T$ characteristics for $\mathrm{NbN}$ films deposited on 4 types of substrates: $\mathrm{Al}_{2} \mathrm{O}_{3}$, $\mathrm{Si}, \mathrm{SiO}_{2}$, and $\mathrm{Si}_{3} \mathrm{~N}_{4}$. The $T_{\mathrm{c}}$ values obtained for films on different substrates varied from $T_{\mathrm{c}}=5.5 \mathrm{~K}$ to $9.5 \mathrm{~K}$. As expected, $T_{\mathrm{c}}$ was most suppressed for the thinnest films. In Fig. 7b one can see the same set of the $R$ vs. $T$ characteristics, but for the annealed films. We observe that the RTP process results in a significant increase of $T_{\mathrm{c}}$ for all our films. For films on $\mathrm{Al}_{2} \mathrm{O}_{3}, \mathrm{Si}$, and $\mathrm{SiO}_{2}$ the $T_{\mathrm{c}}$ values reach the $13-14 \mathrm{~K}$ range. Only for $\mathrm{NbN}$ films deposited on the $\mathrm{Si}_{3} \mathrm{~N}_{4}$ substrate we observed degradation of the superconducting properties, but our post-measurement inspection simply showed that those films cracked during RTP.

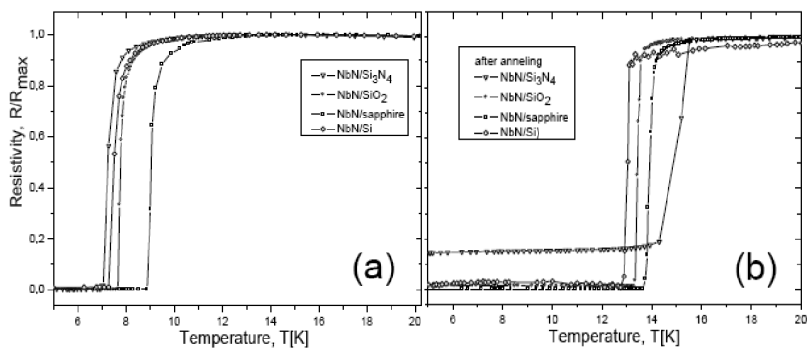

Fig. 7. Dependence of the resistance vs. temperature for $20 \mathrm{~nm} \mathrm{NbN}$ films deposited on sapphire, $\mathrm{Si}(001)$, $\mathrm{SiO}_{2}$ and $\mathrm{Si}_{3} \mathrm{~N}_{4}$ substrates: (a) as-deposited, (b) annealed in $\mathrm{Ar}$ at $1000^{\circ} \mathrm{C}$ for $20 \mathrm{~min}$.

\section{Conclusions}

We have presented our investigations of structural and superconducting transport properties of ultrathin $\mathrm{NbN}$ films deposited on various, electronics and optoelectronics viable substrates. Our active RF sputtering method of fabrication resulted in an excellent crystalline quality (very narrow rocking curve, no mosaics tilt) of the resulting NbN films. The films deposited on sapphire were epitaxial, while the ones on the $\mathrm{Si}, \mathrm{SiO}_{2}$ and $\mathrm{Si}_{3} \mathrm{~N}_{4}$ substrates were textured, but still possessed very good properties. All our samples were characterized with good superconducting properties, with $T_{\mathrm{c}}$ as high as $12.5 \mathrm{~K}$ for $6 \mathrm{~nm}$ thick film deposited on sapphire. We have also demonstrated that the process of rapid annealing improved smoothness of all our thinnest films and increased their $T_{\mathrm{c}}$ 's. We conclude that presented ultrathin $\mathrm{NbN}$ films due to their substrate compatibility have a good potential to be used in SSPD devices integrated with other either electronic or optoelectronic circuitry.

\section{Acknowledgments}

The authors thank I. Zaytseva for her contribution in measurements. This work was supported by the Polish Ministry of Science under project No. 4426/B/TO2/ 2007/33 and by the European Union within European Regional Development Fund, through grant Innovative Economy (POIG.01.01.02-00-108/09, "MIME").

\section{References}

[1] G.N. Gol'tsman, O. Okunev, G. Chulkova, A. Lipatov, A. Semenov, K. Smirnov, B. Voronov, A. Dzardanov, C. Williams, R. Sobolewski, Appl. Phys. Lett. 79, 705 (2001).

[2] K.M. Rosfjord, J.K.W. Yang, E.A. Dauler, A.J. Kerman, V. Anant, B. Voronov, G.N. Gol'tsman, K.K. Berggren, Opt. Expr. 14, 527 (2006).

[3] G. Gol'tsman, O. Minaeva, A. Korneev, M. Tarkhov, I. Rubtsova, A. Divochiy, I. Milostnaya, G. Chulkova, N. Kaurova, B. Voronov, D. Pan, A. Cross, A. Pearlman, I. Komissarov, W. Slysz, R. Sobolewski, IEEE Trans. Appl. Supercond. 17, 246 (2007).

[4] J. Chen, J.B. Altepeter, M. Medic, K.F. Lee, B. Gokden, R.H. Hadfield, S.W. Nam, P. Kumar, Phys. Rev. Lett. 100, 133603 (2008).

[5] M.T. Jarvi, M.J. Niedre, M.S. Patterson, B.C. Wilson, Photochem. Photobiol. 82, 1198 (2006).

[6] S. Tatsuya, S. Shoichi, S. Nami, Y. Tetsuya, J. Ling, U. Yoshinori, M. Hiroyuki, Y. Satoshi, IEEE Trans. Appl. Supercond. 23, 45004 (2010).

[7] F. Marsili, D. Bitauld, A. Gaggero, R. Leoni, F. Mattioli, S. Hold, M. Benkahoul, F. Lévy, A. Fiore, Opt. Expr. 16, 3191 (2008); F. Marsili, D. Bitauld, A. Gaggero, R. Leoni, F. Mattioli, S. Hold, M. Benkahoul, F. Lévy, A. Fiore, Opt. Expr. 16, 3191 (2008).

[8] J.R. Gao, M. Hajenius, F.D. Tichelaar, T.M. Klapwijk, B. Voronov, E. Grishin, G.N. Gol'tsman, C.A. Zorman, M. Mehregany, Appl. Phys. Lett. 91, 062504 (2007).

[9] J.C. Villegier, S. Bouat, P. Cavalier, R. Setzu, R. Espiaude Lamaëstre, C. Jorel, P. Odier, B. Guillet, L. Mechin, M.P. Chauvat, P. Ruterana, IEEE Trans. Appl. Supercond. 19, 3375 (2009).

[10] S. Miki, M. Fujiwara, M. Sasaki, Z. Wang, IEEE Trans. Appl. Supercond. 17, 285 (2007).

[11] R. Espiau de Lamaëstre, Ph. Odier, E. Bellet-Amalric, P. Cavalier, S. Pouget, J.-C. Villegier, J. Phys., Conf. Series 97, 012046 (2008).

[12] M. Kidszun, R. Hühne, B. Holzapfel, L. Schultz, Supercond. Sci. Technol. 23, 025010 (2010). 